\title{
A Mathematical model for Impact of citalopram on the HPA system and combined DEX/CRH test in 30 unipolar depressed patients.
}

\author{
T.Geetha ${ }^{*}$, K.Sathishkumar ${ }^{* * *}$ \\ "Asst.Prof. of Mathematics, Kunthavai Naacchiyar Govt. Arts College for women, (Autonomous), Thanjavur- \\ 613 007.Tamilnadu, India. \\ *Asst.Prof. of Mathematics,Anjalai Ammal-Mahalingam Engineering College, Kovilvenni - 614403. \\ Thiruvarur Dist., Tamilnadu, India.
}

\begin{abstract}
In this paper we introduce bivariate sinh-normal distribution, which has seven parameters. Due to presence of seven parameters it is a very flexible distribution .Different properties of this new distribution has been established. We consider a bivariate model which can be obtained by transforming the sinh-normal distribution and it is a generalization of the bivariate Birnbaum-Saunders distribution. Several properties of the bivariate Birnbaum-Saunders distribution can be obtained as special cases of the proposed generalized bivariate Birnbaum-Saunders distribution. Twenty three patients responded ( $\geq 50 \%$ reduction in the $\mathrm{HDRS}_{21}-$ score), and 17 of them also reached criteria of remission (HDRS $\leq 7$ ). Baseline (dexamethasone suppressed) and CRH stimulated ACTH concentrations significantly decreased from day 0 to day 28. CRH stimulated cortisol concentrations also fell, although not significantly, but baseline cortisol concentrations exhibited a significant increase from day 0 to day 28. Finally we conclude that the medical curve and Mathematical curve for disease control is higher than the probability density functions which are monotonic functions.
\end{abstract}

Key words: ACTH, cortisol, Sinh-normal distribution, bivariate Birnbaum-Saunders distribution.

Mathematical subject AMA classification: $62 \mathrm{H}_{x x} ; 62 \mathrm{NO}_{5} ; 90 \mathrm{~B} 25$.

\section{INTRODUCTION}

Antidepressants are fundamental in the treatment of depression [1]. However, all antidepressants share a nonresponse rate of $30-50 \%$. Recent analyses show that there is no convincing evidence that nonresponders to an antidepressant do benefit from a switch to another antidepressant, as compared to simply continuing the hitherto ineffective antidepressant. This could in part be explained by the fact that almost all antidepressants share comparable neurobiological effects.I.e. Enhancement of the serotonin and norepinephrine neurotransmission in the CNS [2]. Therefore one focus of psychiatric research is to develop agents for the treatment of depression with different modes of action. In this paper, the hypothalamic Pituitary adrenocortical (HPA) system is of great interest, because alterations in depressed subjects and changes to the system under different treatment approaches have repeatedly been demonstrated.

Frequently, the effects of antidepressive compounds on the HPA system have been investigated with the goal of understanding what alterations in the HPA system regulation accompany an improvement in depressive psychopathology. A central hypothesis states that depression is typically associated with a HPA system overdrive, which is cut back in parallel to antidepressive treatment [4]. However, the results were partially inconsistent and hence it appears likely that anti depressive substances as well as subgroups of depression have to be differentiated. Pariante for instance concluded in his recent review that "drugs with opposite effects on the HPA axis have antidepressant effects", and that it is neither conclusive "that high levels of glucocorticoidsalways have a depressiogenic effect, nor that decreasing the effects of these hormones always has an anti depressant effect" [14]. This resulted in the demand for personalized medicine with treatments tailored to the specific pathology of the patients [5]. As a result of this complexity, researchers demanded more studies of the effects of antidepressants on the HPA system

The combined dexamethasone/CRH test (DEX/CRH test) is probably the most sensitive challenge test of the HPA system in psychiatric patients [6]. Only a few studies on the effects of antidepressants with that particular method have been conducted up to now [12], e.g.with tricyclic antidepressants and with mirtazapine In most countries SSRIs represent the most frequently described class of antidepressants. Only two studies up to now have investigated SSRI effects with repeated applications of the DEX/CRH test. [13] 
found a reduction of the ACTH and cortisol response during treatment with citalopram as compared to the pre SSRI situation. The other SSRI studied so far is paroxetine.

We here present the results of a study of 30 unipolar depressed patients, examined with the combined DEX/CRH test right before the start, and after four weeks, of treatment with citalopram. We chose citalopram, because besides its Senantiomer escitalopram, citalopram is the most selective SSRI, acting almost exclusively on the serotonin transporter. This offers the opportunity to focus on serotonergic effects on the HPA system regulation. In addition, we aimed to include mainly drug naive patients (i.e. without previous pharmacotherapy for the index episode) to exclude influences of preceding pharmacological treatment on the HPA system.

\section{MATHEMATICAL MODELS:}

The main aim of this paper is to introduce bivariate sinh-normal (BSHN) distribution, which is a natural extension of SHN distribution of one dimension to two dimensions. Recently, Kundu et al. [9] introduced a bivariate Birnbaum-Saunders distribution. It is observed that the bivariate logBirnbaum-Saunders distribution can be obtained as a

Suppose $\mathrm{Y} \sim \mathrm{SHN}(\alpha, \sigma, \mu)$ then the probability density function [10](PDF) becomes

$$
f_{Y}(y ; \alpha, \sigma, \mu)=\Phi(a(y ; \alpha, \sigma, \mu)) A(y ; \alpha, \sigma, \mu) ; \quad y \in R
$$

Here $\Phi($.$) is the PDF of a standard normal random variable.$

By simple transformation, it follows that if $\mathrm{Y} \sim \operatorname{SHN}(\alpha, \sigma, \mu)$, then

$$
Z=\frac{2}{\alpha} \sinh \left(\frac{y-\mu}{\sigma}\right)-N(0,1)
$$

Here $\mathrm{N}(0,1)$ denotes a standard normal random variable.

Rieck [15] has established the following properties of a SHN distribution. The PDF of SHN is symmetric about the location parameter $\mu$.The distribution is strongly unimodal for $\alpha \leq 2$ and it is bimodal if

$$
\begin{aligned}
& \mathrm{Y} \sim \operatorname{SHN}(\alpha, \sigma, \mu) \text { then } \\
& \frac{2(Y-\mu)}{\alpha \sigma} \rightarrow N(0,1) \text { as } \alpha \rightarrow 0
\end{aligned}
$$

It is an absolute continuous distribution with the PDF[11]

$$
f_{T}(t ; \alpha, \beta)=\frac{1}{2 \sqrt{2 \Pi} \alpha \beta}\left[\left(\frac{\beta}{t}\right)^{\frac{1}{2}}+\left(\frac{\beta}{t}\right)^{\frac{3}{2}}\right] \exp \left[-\frac{1}{2 \alpha^{2}}\left(\frac{t}{\beta}+\frac{\beta}{t}-2\right)\right], t>0
$$

Here $\alpha$ is the shape parameter and $\beta$ is the scale parameter.

A standard BSHN random variable has the PDF which is centred at $(0,0)$ and it is symmetric around $(0,0)$.It is observed that the surface of the PDF can be unimodal,bimodal or multimodal depending on the parameter values.
$\alpha>2$.It can be easily seen using L'Hospital's rule, that if

\section{APPLICATIONS:}

\section{1 АCTH}

Mean baseline ACTH (before CRH injection) declined from $14.9 \mathrm{pg} / \mathrm{ml}$ before citalopram to $9.4 \mathrm{pg} / \mathrm{ml}$ after 4 weeks of treatment ( $\mathrm{p}<0.001$, Wilcoxon's matched pairs test). With regard to the ACTH values after $\mathrm{CRH}$ stimulation, a significant decrease during treatment was observed for the total AUC as well (from 2077 to 1393, p = 0.001). 
However, net AUC did not change significantly $(\mathrm{p}=$ 0.73 ), indicating that the main change during treatment refers to an increased suppression effect to dexamethasone, whereas the response to the $\mathrm{CRH}$ stimulus was comparable between day 0 and day 28 .

\subsection{CORTISOL}

Regarding cortisol, a significant increase in the mean baseline values (before CRH injection) was observed (from $14.5 \mathrm{ng} / \mathrm{ml}$ to $21.9 \mathrm{ng} / \mathrm{ml}, \mathrm{p}=0.03$, Wilcoxon's matched pairs test), indicating a reduction of the suppressive effect of dexamethasone on the cortisol concentration during treatment with citalopram. The CRH stimulated values (total AUC, net AUC) remained unchanged.

\subsection{CORTISOL/ACTH RATIO}

The cortisol/ACTH ratio is a marker of adrenal cortex responsiveness, indicating the amount of cortisol that is produced in response to a defined quantity of ACTH. According to the fact that the ACTH parameters decreased while the cortisol parameters tended to increase, a significant increase in that ratio was detected for the baseline ratio and for the total ratio $(\mathrm{p}=0.00, \mathrm{p}=0.02$ respectively), indicating an increased responsiveness of the adrenal gland to ACTH. Again, the net ratio did not change significantly $(\mathrm{p}=0.15)$, indicating that the main changes from day 0 to day 28 concern the suppressive effect of dexamethasone.
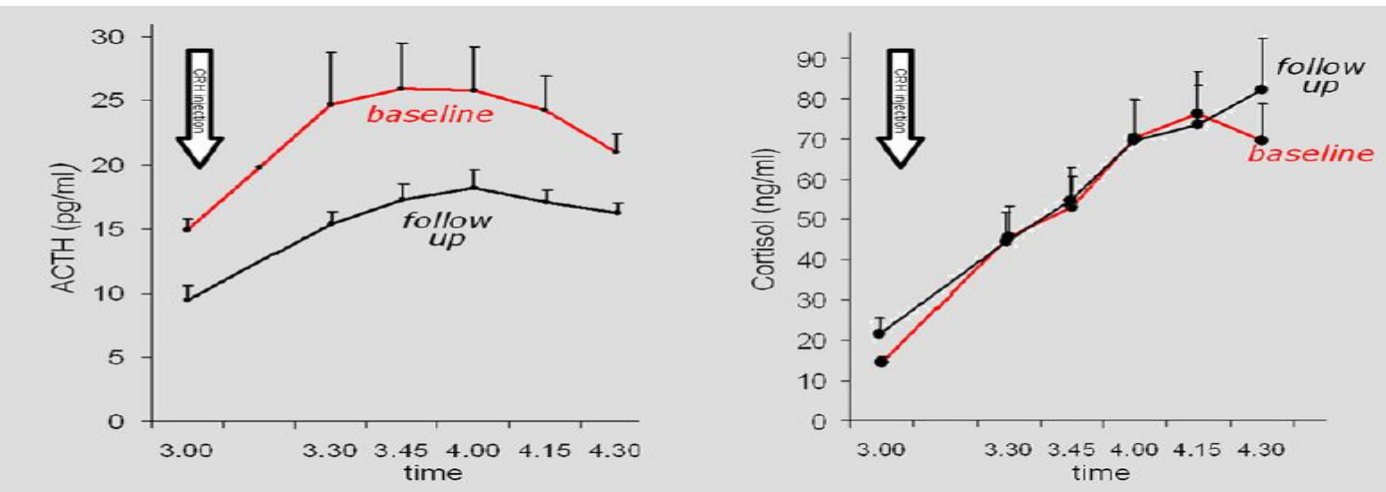

Fig 3.1(a)ACTH and Cortisol response (mean concentrations \pm SEM) in the combined DEX/CRH test on the day before citalopram treatment (baseline) and after four weeks of treatment (follow-up) $(\mathrm{N}=30)$

\section{DISCUSSION}

Thirty well characterized, mostly drug naive patients participated in our study. With regard to $\mathrm{ACTH}$, the serum concentrations in the combined dexamethasone/CRH test decreased after four weeks of treatment with the SSRI citalopram. This is in line with what has predominantly been observed during antidepressant treatment in general and with what has been observed in the two studies with repeated DEX/CRH tests in SSRI treated patients conducted so far. However, it is noteworthy that this decrease particularly seems to be attributed to an improved suppressive effect of dexamethasone. This is indicated by the fact that only the baseline values (3 p.m., i.e. before CRH stimulation) and the total AUC values, but not the net AUC values decreased significantly from day 0 to day 28 .

Regarding cortisol, the stimulating effect of CRH decreased from day 0 to day 28 of citalopram treatment indicated by a fall of net AUC from 3449 to $2932(\mathrm{p}=0.4)$. Again, this is consistent with results from other antidepressants .However, the baseline cortisol concentrations ( 3 p.m.) increased significantly during treatment, denoting that the suppressive effect of dexamethasone declined.These findings indicate that a blunting effect of the citalopram treatment in the DEX/CRH test was only present on the pituitary level of the HPA system, whereas on the level of the adrenal gland a partial activation was found, besides the good clinical efficacy of citalopram in our study. Typically, treatment with antidepressants and improvement in depressive symptoms tends to be associated with a blunting of the HPA system response. However, the effect of antidepressants on the HPA system seems to be temporarily associated with the activating effects of most antidepressants in the beginning of the treatment .In addition, recent findings and reviews have pointed out that an activation of that system by other substances trialed for treatment of depression atleast does not hinder the relief of depressive symptoms. [12] in their recent review concluded that effective antidepressive pharmaceuticals might alternatively lead to an activation or a blunting of cortisol regulation, or even both. Their metaanalysis revealed that approximately $56 \%$ of depressed patients did not exhibit a decrease in their cortisol concentration during treatment .Our group found a HPA system activating effect in lithium augmentation and in lithium monotherapy, despite a good antidepressive outcome.

The increase in baseline cortisol (prior to CRH injection) from day 0 to day 28 contrasts with 
the drop in baseline ACTH. Since cortisol is released by the adrenal gland primarily due to stimulition by ACTH, alterations of the adrenal cortex have to be taken into account. As also indicated by the significant rise of the cortisol/ ACTH ratio from day 0 to day 28 , an increase of the sensitivity of the adrenal cortex has to be considered.A direct effect of SSRIs on the adrenal gland has not been described up to now. However, alterations of that gland associated with a depressive episode and its regression have repeatedly been reported. Imaging studies have shown that the volume of the adrenal gland is enlarged in depression (with a normalization after recovery), presumably induced by excessive ACTH stimulation. This continued overstimulation is paralleled by an increase in the sensitivity of the adrenal cortex to $\mathrm{ACTH}$, resulting in a rise in the cortisol/ACTH ratio. Depressed subjects exhibit a greater cortisol response to the same amount of ACTH than normal control subjects [8]. After CRH stimulation, depressed subjects, in comparison to healthy controls, repeatedly only showed an altered ACTH response, but a similar cortisol response [3] also indicating a modified ACTH response by the adrenal gland. A low cortisol/ACTH ratio has been associated with a response to lithium augmentation. These facts point out that the responsiveness of the adrenal gland to ACTH is regulated and flexible, which includes the possibility that antidepressants directly or indirectly have an impact on it. However, due to the study design, our results unfortunately cannot further elucidate this aspect.

With only 7 nonresponders, a comparison with the 23 responders has methodological limitations. Responders and nonresponders to the citalopram treatment did not significantly differ with regard to their DEX/CRH test results, neither before, nor after 28 days of treatment. This indicates that the DEX/CRH test was not helpful in predicting response to the treatment. That means that, besides the fact that this endocrinological test is rather too complex for an everyday routine, the test does not provide adequate support for a decision for or against citalopram treatment. This is in line with other studies on the predictive value of the DEX/ CRH test. However, previous studies suggesting a predictive effect of the DEX/CRH test reported a time lag between the restoration of the HPA axis function and psychopathological improvement. For instance, [7] showed that a reduced cortisol response to the DEX/CRH test is not associated with the current depression state but with psycho pathological improvement two to three weeks later. A number of studies performing the DEX/CHR test in remitted depression showed a predictive effect for relapse during a follow up period of several months.

In addition, the two studies conducted so far to prospectively investigate SSRI effects on the
DEX/CRH test included subjects who had failed to respond to at least one antidepressant medication trail for the current episode .It might well be that treatment resistance and a more pronounced depression severity on the one hand, and pretreatment with antidepressants on the other hand, lead to changes in the cortisol regulation differing to some extent from those seen in the subjects of the study presented here. The most pronounced alterations in the HPA system were found in patients with severe depression of the melancholic or psychotic subtype. With 20 and 22 patients on SSRI respectively, these two studies were somewhat smaller than the study presented here $(\mathrm{N}=30)$. Another reason for the small cortisol effects in the current study might be related with the sample selection, as it is well known that not all patients with depression show an abnormal HPA axis regulation, especially in an unselected sample of depressed outpatients .In addition, with a mean of $43.8( \pm 13.8)$ years, the subjects reported here were younger than the depressive patients in most of the preceding studies applying the DEX/CRH test, which were on average between 50 and 69 years old. [13] studied younger patients (mean: $36 \pm 9.6$ years). Higher age is associated with a more pronounced alteration of the HPA system in depression. As with nearly every study, this paper has methodological strengths and limitations. On the positive side a small drop out rate, a good characterization of the included sample and an only minor proportion of participants with pharmacological pretreatment for their depressive index episode (17\%) can be mentioned. Established standardized clinical interviews and rating scales were used to monitor and validate their depressive symptoms. The neuroendocrinological results were obtained by the best established and validated test in this field, the combined DEX/CRH test. With one exception, patients were not hospitalized, thereby representing the majority of patients treated with SSRIs. On the other hand, we did not include a control group. A placebo component would have been especially necessary to reliably interpret the clinical efficacy of the citalopram treatment and to evaluate if the participants had indeed shown an overactive HPA system before the start of treatment. However, this was not the main objective of our study, which primarily focused on the changes in cortisol regulation induced by citalopram. Because the start of citalopram was the only systematic change between the first and the second DEX/CRH test, it is rather unlikely that the differences found are due to other factors than the SSRI and its therapeutic effects. In addition, the time of the second DEX/CRH test could be a matter of discussion. It cannot be excluded that after a treatment period longer than four weeks a decrease not only in ACTH, but also in the cortisol parameters might have been demonstrated. 


\section{MATHEMATICAL RESULTS}

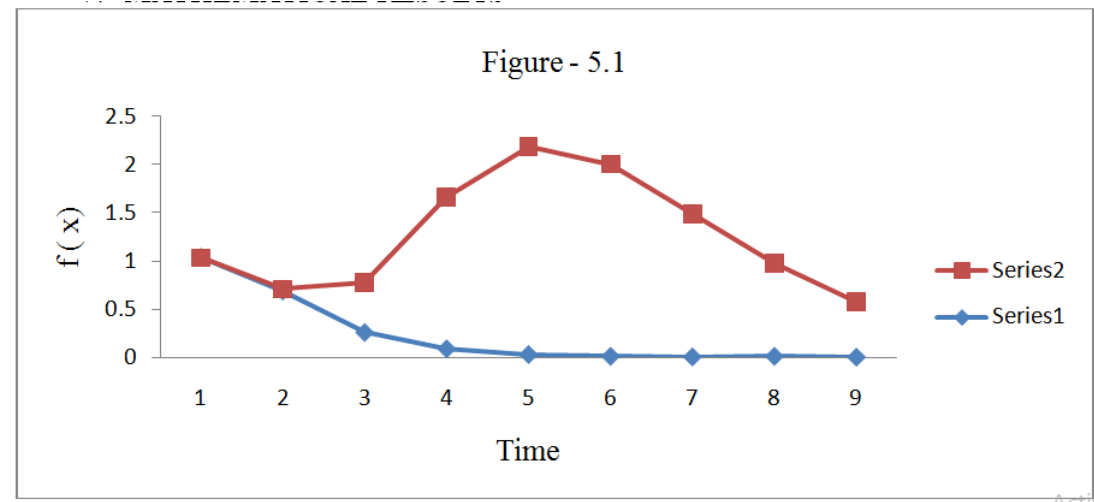

\section{CONCLUSION}

The blunting of the ACTH response in the DEX/CRH test under citalopram is in line with what has been observed in most studies with antidepressants. However, the partial rise in cortisol concentrations indicates an increase in the sensitivity of the adrenal cortex to ACTH. State dependent alterations in the volume and the ACTH responsiveness of the adrenal gland have repeatedly been reported in depressed subjects, which indicate the possibility that SSRIs such as citalopram might exhibit a direct or indirect effect on the adrenal cortex. Twenty three patients responded $(\geq 50 \%$ reduction in the $\mathrm{HDRS}_{21}-$ score), and 17 of them also reached criteria of remission (HDRS $\leq 7$ ). Baseline (dexamethasone suppressed) and $\mathrm{CRH}$ stimulated ACTH concentrations significantly decreased from day 0 to day 28. CRH stimulated cortisol concentrations also fell, although not significantly, but baseline cortisol concentrations exhibited a significant increase from day 0 to day 28. Finally we conclude that the medical curve and Mathematical curve for disease control is higher than the probability density functions which are monotonic functions.

\section{REFERENCES}

[1] Bauer M, Bschor T, Pfennig A, Whybrow PC, Angst J, Versiani M, et al(2007).

World Federation of Societies of Biological Psychiatry (WFSBP) Guidelines for

Biological treatment of unipolar depressive disorders in primary care. World Journal

Of Biological Psychiatry;8:67-104.

[2] Berton O, Nestler EJ. (2006)New approaches to antidepressant drug discovery: beyond monoamines. Nature Reviews. Neuroscience; 7:137-51.

[3] Gold PW, Loriaux DL, Roy A, Kling MA, Calabrese JR, Kellner CH, et al (1986) Responses to corticotropin-releasing hormone in the hypercortisolism of depression and Cushing's disease. Pathophysiologic and diagnostic implications. New England Journal of Medicine;314:1329-35.

[4] Holsboer F. The corticosteroid receptor hypothesis of depression. (2000). Neuropsychopharmacology;23:477-501.

Holsboer F, Barden N.( 1996). Antidepressants and hypothalamic pituitary adrenocorticalregulation. Endocrine Reviews; 17:187-205.

[5] Holsboer F, Ising M(2010). Stress hormone regulation: biological role and translation into therapy. Annual Review of Psychology;61:81109. C1-11.

[6] Ising M, Kunzel HE, Binder EB, Nickel T, Modell S, Holsboer F(2005). The combined dexamethasone/CRH test as a potential surrogate marker in depression. Progress in Neuro-Psychopharmacology \& Biological Psychiatry; 29: 1085-93.

[7] Ising M, Horstmann S, Kloiber S, Lucae S, Binder EB, Kern N, et al(2007). Combined dexa- methasone/corticotropin releasing hormone test predicts treatment response in major depression e a potential biomarker? Biological Psychiatry;62:47-54.

[8] Jaeckle RS, Kathol RG, Lopez JF, Meller WH, Krummel SJ(1987). Enhanced adrenal sensitivity to exogenous cosyntropin (ACTH alpha 1-24) stimulation in major depression. Relationship to dexamethasone suppression test results. Archives of General Psychiatry;44:233-40.

[9]

Kundu,D.,Balakrishnan,N.andJamalizadeh,A(2 010).'Bivariate Birnbaum-Saunders distribution and its associated inference", Journalof Multivariate Analysis,; vol.101,113125.

[10] Lakshmi.S and Manickam.A. "Entropy-based measure of uncertainty for growth hormone secretary burst and amplitude during a two-day fast in normal men" The Journal of the Indian Academy of Mathematics. Vol.35 Number.2, 
2013. Pp.341-358. ISSN: 0970-5120. Indexed in "Zentralblatt Math Database" (Zbl pre 06342181).

[11] Dr.S.Jayakumar and Mrs.G.Ramya Arockiamary and Mr.A.Manickam

"A Mathematical Model for Role of Gemcitabine and Carboplatin in Intensively Pretreated Patients with Metastatic Breast Cancer using Bivariate Sinh-Normal Distribution Model", International Journal of Innovation in Science and Mathematics, Vol .3,Issue 2,Mar 2015

[12] McKay MS, Zakzanis KK(2010). The impact of treatment on HPA axis activity in unipolar major depression. Journal of Psychiatric Research;44:183-92.

[13] Nikisch G, Mathe AA, Czernik A, Thiele J, Bohner J, Eap CB, et al(2005). Long-term citalopram administration reduces responsiveness of HPA axis in patients with major depression: relationship with $\mathrm{S}$ citalopram concentrations in plasma and cerebrospinal fluid (CSF) and clinical response.

Psychopharmacology (Berl);181:751-60.

[14] Pariante CM (2009). Risk factors for development of depression and psychosis. Glucocor ticoid receptors and pituitary implications for treatment with antidepressant and glucocorticoids. Annals of the New York Academy of Sciences; 1179:144-52.

[15] RieckJ.R.Statistical analysis for the BirnbaumSaunders fatigue life distribution Ph.D.thesis(1989),ClemsonUniversity, Department of Mathematical Science ,Canada. 